\title{
Laboratory Tests of Multiplex Detection of PCR Amplicons using the Luminex 100 Flow Analyzer
}

\author{
K. S. Venkateswaran, S. Nasarabadi and R. G. Langlois
}

\section{May 5, 2000}

U.S. Department of Energy

Lawrence

Livermore

National

Laboratory 


\section{DISCLAIMER}

This document was prepared as an account of work sponsored by an agency of the United States Government. Neither the United States Government nor the University of California nor any of their employees, makes any warranty, express or implied, or assumes any legal liability or responsibility for the accuracy, completeness, or usefulness of any information, apparatus, product, or process disclosed, or represents that its use would not infringe privately owned rights. Reference herein to any specific commercial product, process, or service by trade name, trademark, manufacturer, or otherwise, does not necessarily constitute or imply its endorsement, recommendation, or favoring by the United States Government or the University of California. The views and opinions of authors expressed herein do not necessarily state or reflect those of the United States Government or the University of California, and shall not be used for advertising or product endorsement purposes.

Work performed under the auspices of the U.S. Department of Energy by the University of California Lawrence Livermore National Laboratory under Contract W-7405-Eng-48.

This report has been reproduced directly from the best available copy.

Available to DOE and DOE contractors from the Office of Scientific and Technical Information

P.O. Box 62, Oak Ridge, TN 37831

Prices available from (423) 576-8401 http://apollo.osti.gov/bridge/

Available to the public from the National Technical Information Service

U.S. Department of Commerce 5285 Port Royal Rd., Springfield, VA 22161 http://www.ntis.gov/

$\mathrm{OR}$

Lawrence Livermore National Laboratory Technical Information Department's Digital Library http://www.llnl.gov/tid/Library.html 


\title{
LABORATORY TESTS OF MULTIPLEX DETECTION OF PCR AMPLICONS USING THE LUMINEX 100 FLOW ANALYZER
}

\author{
Kodumudi S. Venkateswaran, Shanavaz Nasarabadi and Richard G. Langlois \\ Biology and Biotechnology Research Program \\ Lawrence Livermore National Laboratory \\ Livermore, CA 94551
}

May 5,2000

\section{Introduction}

Lawrence Livermore National Laboratory (LLNL) demonstrated the power of flow cytometry in detecting the biological agents simulants at JFT III. LLNL pioneered in the development of advanced nucleic acid analyzer (ANAA) for portable real time identification. Recent advances in flow cytometry provide a means for multiplexed nucleic acid detection and immuno assay of pathogenic microorganisms. We are presently developing multiplexed immuno assays for the simultaneous detection of different simulants. Our goal is to build an integrated instrument for both nucleic acid analysis and immuno detection. In this study we evaluated the Luminex LX 100 for concurrent identification of more than one PCR amplified product. ANAA has real-time Taqman fluorescent detection capability for rapid identification of field samples. However, its multiplexing ability is limited by the combination of available fluorescent labels. Hence integration of ANAA with flow cytometry can give the rapidity of ANAA amplification and the multiplex capability of flow cytometry. Multiplexed flow cytometric analysis is made possible using a set of fluorescent latex microspheres that are individually identified by their red and infrared fluorescence. A green fluorochrome is used as the assay signal. Methods were developed for the identification of specific nucleic acid sequences from Bacillus globigii $(\mathrm{Bg})$, Bacillus thuringensis $(\mathrm{Bt})$ and Erwinia herbicola (Eh). Detection sensitivity using different reporter fluorochromes was tested with the $L X 100$, and also different assay formats were evaluated for their suitability for rapid testing. A blind laboratory trial was carried out December 22-27, 1999 to evaluate bead assays for multiplex identification of $\mathrm{Bg}$ and $\mathrm{Bt}$ PCR products. 
This report summarizes the assay development, fluorochrome comparisons, and the results of the blind trial conducted at LLNL for the laboratory evaluation of the LX 100 flow analyzer.

\section{Materials and Methods}

\section{Bacterial cultures and spores}

A preparation of purified $\mathrm{Bg}$ and $\mathrm{Bt}$ spores was used for assay development and to provide positive controls for this study. Culturing $\mathrm{Bg}$ in sporulation media produced $\mathrm{Bg}$ spores. Cheryl Strout (LLNL) provided the Bt spore paste. The spore paste was purified as follows. The spore suspension was washed three times with water by vortexing the spore suspension and centrifuging at $5800 \times \mathrm{g}$. The spore paste was then resuspended in $100 \mathrm{mM}$ EDTA / $50 \mathrm{mM} \mathrm{NaCl}, \mathrm{pH} 6.9$ and incubated with $1 \mathrm{mg} / \mathrm{ml}$ lysozyme at $30^{\circ} \mathrm{C}$ for 90 minutes. The suspension was centrifuged, washed two more times, resuspended in 5\% Triton X-100 and sonicated for a minute. The suspension was layered on top of a $20 \%, 40 \%, 60 \%$ Percoll gradient and centrifuged at $772 \times \mathrm{g}$ for fifteen minutes. The $60 \%$ gradient was collected and the spores centrifuged and washed twice with water and finally resuspended in water. Final spore concentrations were determined using a Becton Dickinson FACScan flow cytometer in an immuno assay using fluorescent labeled antibody and was confirmed using plate counts of viable spores. Additional bacterial standards used in some experiments included vegetative Eh cells. All of the $\mathrm{Bg}$ and Eh preparations were derived from Dugway stocks and were quantified by flow cytometric assay as described above.

\section{Fluorescent Microspheres}

Different sets of carboxylated ( $\mathrm{COOH}-)$ and streptavidin coated fluorescent microspheres (LumAvidin) were procured from Luminex Corp. Austin TX.

\section{Oligonuleotides}

Oligonucleotides that were 5' amine-labeled, 5' biotin-labeled, or 3' fluorescent labeled with Cyanine 3 (Сy3) or BODIPY Tetramethyl rhodamine (BODIPY-TMRX) were purchased from either KeyStone Labs, Camarilo, CA or from Oligo's Etc, Portland, OR. The probe sequences of the oligonucleotides used in this study are given below. Probes labeled " $T$ " correspond to the sequence used for our standard Taqman assays, while those labeled "AT" correspond to the complement of the Taqman sequence. 


\begin{tabular}{|c|c|}
\hline $\begin{array}{l}\text { BG238T } \\
\text { BG238AT }\end{array}$ & $\begin{array}{l}\text { 5'-act gaa cag ctg atc gag aca gct gt-3' } \\
5^{\prime} \text {-aca gct gtc tcg atc agc tgt tca gt-3' }\end{array}$ \\
\hline CRYIV182T & $5^{\prime}-$ aaa cca ggt gga atg cca cg-3' \\
\hline CRY IV 182 AT & $5^{\prime}-\operatorname{cgt}$ ggc att cca cct ggt tt-3' \\
\hline EH512T & $5^{\prime}-\mathrm{ccg}$ ggc ttg aac ccc act cc-3' \\
\hline EH512AT & $5^{\prime}$-gga gtg ggg ttc aag ccc gg-3' \\
\hline
\end{tabular}

\section{Fluorescent labeled Reporter}

Streptavidin labeled with Phycoerythrin (PE) or Cy3 were purchased from Caltag Laboratories, Burlingame, CA and streptavidin labeled with Alexa 532 or Alexa 546 were from Molecular Probes, Eugene, OR.

\section{Covalent coupling of capture-oligonucleotides to $\mathrm{COOH}$-Microspheres}

Amino modified oligonucleotides were covalently coupled to carboxylated micropshreres $\left(2.5 \times 10^{6}\right.$ microspheres in $80 \mu \mathrm{l}$ of $0.1 \mathrm{M}$ 2-[N-morpholino] ethanesulfonic acid (MES) (Sigma, St. Louis, MO)) by mixing with the oligos ( 5 nmoles in $5 \mu$ of 0.1 MES, pH 4.5). At two separate times $25 \mu \mathrm{g}$ of freshly made 1-ethyl-3-(3dimethylaminopropyl) carbodiimide hydrochloride (EDC) (Pierce, Rockford, IL) were added to the microsphere mixture; at the beginning of the incubation, and then after 30 minute period. The reaction was occasionally mixed and sonicated during the incubation for 60 minutes at room temperature to keep the microspheres unclumped and in suspension. After coupling, the microspheres were washed in $1 \mathrm{ml}$ phosphate buffered saline containing $0.02 \%$ Tween 20 (Sigma, St. Louis, MO) and centrifuged in a micro centrifuge at $13000 \mathrm{rpm}$ for 2 minutes. The beads were resuspended in phosphate buffered saline containing $0.1 \%$ sodium dodecyl sulfate (SDS), mixed well and centrifuged as mentioned above. The beads were finally resuspended in $0.1 \mathrm{MMES}$ and stored protected from light till further use at $2-8^{\circ} \mathrm{C}$.

\section{Coupling of biotinylated oligonucleotides to LumAvidin Microspheres}

$1 \times 10^{5}$ LumAvidin modified microspheres were resuspended in $125 \mu$ l of phosphate buffered saline containing bovine serum albumin (BSA), pH 7.3, and mixed with $125 \mu \mathrm{l}$ of biotin labeled oligonucleotide $(250 \mathrm{pmol})$. The microsphere mixture was protected from light and incubated at room temperature with shaking. The microspheres were 
centrifuged at $13000 \mathrm{rpm}$ for 2 minutes and washed three times with phosphate buffered saline containing BSA. The microspheres were resuspended in PBS-BSA and stored refrigerated.

\section{Analysis of fluorescent Microspheres}

Fluorescent microspheres were analyzed using the Luminex 100 (LX100) flow analyzer system. It is a compact analysis unit consisting of an analyzer, a Pentium processor personal computer, and the Luminex software. The Luminex 100 individually analyzes microspheres in a flow stream. Each microsphere is accurately classified to its own subset, based on its fluorescent signature. In addition, the Luminex 100 scans each bead for the presence of a reporter fluorescence that quantifies the assay at the bead's surface. The LX100 used in the study is pictured in Fig. 1.

\section{Multiplex Flow Microsphere Oligonucleotide Assay}

\section{Direct Assay}

$10 \mu$ l of capture oligonucleotide coupled LumAvidin or carboxylated microspheres (1000 to 5000 of each microsphere type) were mixed with $40 \mu$ of $1 \mathrm{X}$ hybridization buffer [5.0M tetramethyl ammonium chloride, 0.15\% SDS, $75 \mathrm{mM}$ Tris pH 8.0, $3.0 \mathrm{mM}$ EDTA pH 8.0). $4 \mu \mathrm{l}$ of varying concentrations of fluorescent-labeled reporter oligonucleotides (Cy3 or BODIPY TMRx [BDY)] was added to the hybridization buffer. The mixture was incubated at $55^{\circ} \mathrm{C}$ for 15 minutes. $150 \mu$ of hybridization buffer was added to the bead mixture, and $50 \mu$ of that sample was analyzed in the instrument without any washing of the microspheres. In the wash format, the mixture was centrifuged at $13000 \mathrm{rpm}$ for 2 minutes, the supernatant removed and the microspheres resuspended in $100 \mu \mathrm{l}$ of $1 \mathrm{x}$ hybridization buffer. Microspheres were washed again and resuspended in $200 \mu \mathrm{l}$ of hybridization buffer. $50 \mu$ of the suspension analyzed in $L X 100$ flow analyzer.

\section{Indirect Assay}

$10 \mu \mathrm{l}$ of capture oligonucleotide coupled carboxylated microspheres (1000 to 5000 of each microsphere type) were mixed with $40 \mu \mathrm{l}$ of $1 \mathrm{X}$ hybridization containing $4 \mu \mathrm{l}$ of varying concentrations of biotinylated oligonucleotides. The mixture was incubated at $55^{\circ} \mathrm{C}$ for 15 minutes. The mixture was centrifuged at $13000 \mathrm{rpm}$ for 2 minutes, the supernatant removed and the microspheres resuspended in $100 \mu \mathrm{l}$ of $1 \mathrm{x}$ hybridization buffer containing $1 \mu \mathrm{g} / \mathrm{ml}$ of Streptavidin labeled with either PE or Cy3 or Alexa 546 or Alexa 532. The microspheres were incubated at $55^{\circ} \mathrm{C}$ for 15 minutes. The mixture is washed twice as mentioned before and resuspended in $200 \mu \mathrm{l}$, of which $50 \mu \mathrm{l}$ 
analyzed in the LX 100 flow analyzer. In some experiments, samples from the indirect assay were also analyzed using a conventional flow cytometer [Becton Dickinson (BD) FACScan] for comparison of the results with the $L X 100$ analysis.

\section{Samples for the LLNL blind laboratory trial}

A total of 24 coded samples were used for the blind laboratory trial. The following sample types were prepared for PCR amplification as part of this trial: 6 samples with no template (no template controls, NTC); 6 samples containing B.g. spores at concentrations ranging from $10^{3}$ to $10^{6}$ per $\mathrm{ml} ; 6$ samples containing B.t. spores at concentrations ranging from $10^{3}$ to $10^{6}$ per $\mathrm{ml}$; and 6 samples containing B.g. and B.t. at concentrations ranging from $10^{4}$ to $10^{6}$ per $\mathrm{ml}$. The samples were transferred to random numbered tubes by a scientist not involved with the analysis to ensure a blind trial. The description of the samples and their identification \# used in the blind test are given in Table 1. Inferred identities for each sample were recorded after the completion of PCR amplification and multiplex bead assays before the samples were decoded to reveal their true identities.

\section{Polymerase Chain Reaction (PCR) Amplification}

The primer and probe sequences utilized in this study target Bt CRY IVa gene yielding an amplified product size of $80 \mathrm{bp}$ and target $\mathrm{Bg}$ yielding an amplified product of $\sim 280$ bp. The PCR mix consisted of 1 x PCR buffer (Life Technologies), $6 \mathrm{mM} \mathrm{MgCl}_{2}$ (Sigma), $0.2 \mathrm{mM}$ each dATP, dCTP, dGTP, (Boehringer Mannheim), $0.4 \mathrm{mM}$ dUTP (Boehringer Mannheim), $0.04 \mu \mathrm{M}$ each primers (Synthegen) and $0.25 \mathrm{U}$ Platinum Taq polymerase (Life Technologies). PCR amplification was performed using a Perkin Elmer 9600 thermal cycler in a reaction volume of $50 \mu \mathrm{l}(10 \mu \mathrm{l}+40 \mu \mathrm{l}$ mix $)$. Cycling parameters were $96^{\circ} \mathrm{C}$ for 1 minute and then 50 cycles of $95^{\circ} \mathrm{C}$ for 15 seconds and $60^{\circ} \mathrm{C}$ for 1 minute. PCR amplified products were tested using an agarose gel with ethidium bromide staining and visualized in an UV transilluminator. Gel analysis confirmed the presence of the appropriate PCR amplicons, or lack of product after amplification, for each of the laboratory trial samples.

Competitive inhibition multiplex flow microsphere oligonucleotide assay of PCR amplified products for the laboratory trial conducted at LLNL

$5 \mu \mathrm{l}$ of a mixture of two biotinylated oligonucleotides BgT ( 20 fmoles) and BtT (5 fmoles) was mixed with $30 \mu$ of $1 \times$ hybridization buffer containing TMAC. $5 \mu \mathrm{l}$ of the PCR amplified product was then added and the mixture kept at $98^{\circ} \mathrm{C}$ for 10 minutes, followed 
by $45^{\circ} \mathrm{C}$ for 5 minutes. $15 \mu$ of a mixture of 3 microspheres (2000 microspheres each of C153BgAT, C152EhT and C158BtAT) was added and incubated at $45^{\circ} \mathrm{Cfor} 15$ minutes. $10 \mu$ l of streptavidin PE (100 ng per tube) was then mixed with the sample and incubated at room temperature for 5 minutes. The mixture is centrifuged at $13000 \mathrm{rpm}$ for 2 minutes and the supernatant was discarded. The microspheres were resuspended in $125 \mu$ of $1 \times$ hybridization buffer, and $50 \mu$ was analyzed in the $L X$ 100.

\section{Results}

\section{Multiplex bead analysis in LX100 flow analyzer}

During the course of this study Luminex has produced three different sets of multiplex microspheres, each set adapted to different hardware and software upgrades to the LX 100 instrument. While each of these upgrades have improved instrument performance, they have had the short-term disadvantage that assays prepared with one bead set become obsolete and must be re-done with new bead sets after each modification of the instrumentation. The first bead sets were the beta testing beads that came as a set of 9 beads corresponding to 9 specific regions in the two dimensional bead classification dot plot. Our initial experiments were done with four sets of beta test beads, two each for $\mathrm{Bg}$ and $\mathrm{Eh}$ with complementary sequences. Results of a duplex assay for $\mathrm{Bg}$ and $\mathrm{EH}$ PCR products by competitive assay are shown in Fig.2. It is clear from the data shown in Fig.2, that the reporter signal varied with the oligonucleotide sequence, and with the microsphere type. The inhibition of the fluorescent reporter is more pronounced with the Eh sequence compared with the $\mathrm{Bg}$ sequence. However, the inhibition was specific for the respective amplified product present during hybridization.

\section{Effect of washing on the oligonucleotide binding assay}

Washing the microspheres can reduce non-specific binding of the fluorescent- labeled reporter and increase the specific signal intensity. Washing is also useful in getting rid of the excess fluorochromes in the solution that can interfere in some cases with the measurement. Results from the direct binding assays for $\mathrm{Bg}$ and $\mathrm{Eh}$ done with and without a wash step are shown in Fig. 3. Varying oligonucleotide concentrations were tested, and the results are shown as titration curves with mean fluorescence intensity on the $\mathrm{Y}$-axis versus the oligonucleotide concentration on the $\mathrm{X}$-axis. It is evident from the titration curves that washing can reduce non-specific fluorescent binding observed on the microspheres in the range of 2 to 10 fold at different probe concentrations. It is 
also clear from the data that the limit of detection is better with washing, and that washing improves signal to noise ratio.

\section{Comparison of the Luminex 100 Analyzer with the BD FACScan}

The BD FACScan flow cytometer has the capability to analyze five parameters (forward scatter, side scatter and three fluorescence channels) using a single laser excitation. The Luminex 100 analyzer uses two lasers, and measures four parameters (doublet discrimination, and three fluorescence channels for classifier 1, classifier 2 and reporter). We compared the performance of fluorescent microsphere analysis on both flow cytometers, and the results are shown in Fig. 4. Streptavidin PE was used as the fluorescent label. Carboxylated microspheres coated with capture oligos specific for $\mathrm{Bg}$ and $\mathrm{Eh}$, and complementary biotinylated oligonucleotides, were used for this study. The LX 100 analysis of microspheres in the indirect assay gave mean fluorescent intensity values very similar to those seen with the FACScan analysis. The data from the samples with or without wash are comparable with both instruments, and the usefulness of washing is clearly evident from the lower detection limit and increased signal to noise ratio.

\section{Evaluation of different fluorescent labeled oligonucleotides with streptavidin and carboxylated microspheres}

Oligonucleotides directly labeled with Cy3 or BODIPY-TMRx were tested as reporter molecules in the microsphere assay. Both these dyes can be excited at $532 \mathrm{~nm}$ and emit fluorescence around $575 \mathrm{~nm}$ that can be detected using the photomultiplier tube in the LX 100. Reporter oligonucleotides directly labeled with fluorescent molecules are advantageous over the use of streptavidin labeled PE because they are small in size and non-specific binding can be low. These oligos can easily be used on streptavidin coated microspheres having biotinylated capture oligonucleotides. We also evaluated the performance of different fluorochromes (Cy3, Alexa 546 and Alexa 532, PE) attached streptavidin as reporter for the detection of biotinylated oligonucleotides. Experiments were done to calculate the signal to noise ratio of these different sets and also to find out the effect of wash steps in improving the limit of detection. Results of different experiments are summarized in Table 2, where the mean fluorescent intensity (MFI) of positive microspheres is given. The level of non-specific binding of the fluorescent oligonucleotides to the microspheres is shown in the column "Meanother/no dye". These values correspond to the mean fluorescence of beads exposed to a non-complementary fluorescent reporter divided by the mean intensity of beads 
with no added fluorescence reporter. It is clear from the data that the non-specific binding of fluorescent reporter is high in the sets where there was no wash step involved in the assay. Washing the microspheres considerably decreased the nonspecific binding of the fluorescent reporter onto microspheres. Streptavidin labeled with PE gave the brightest staining of the microspheres, where as the small molecular weight fluorescent dyes as labels gave 2- to 3-fold lower signal.

Results of the microsphere analysis using LumAvidin and carboxylated microspheres with in the direct binding assay with and without washing are given in Table 3 . The "signal/noise" values in this and subsequent tables was calculated from the mean intensity of beads hybridizing with the reporter probe divided by the mean intensity of beads that do not hybridize with the reporter probe. LumAvidin beads gave brighter signals with both Cy 3 and BODIPY-TMRx labeled oligos compared with carboxylated beads. The approximately two-fold decrease in signal observed with carboxylated beads might be due to the quenching of fluorescence signal because of the acidic $\mathrm{pH}$ at the surface of the carboxylated beads. BODIPY-TMRX showed slightly higher fluorescent signal than Сy3, however it also showed higher non-specific binding, and hence had a lower signal to noise ratio than Cy3. The best signal noise ratio $(\sim 66)$ was seen with Cy3 and LumAvidin beads.

Testing of different fluorescent labeled streptavidins was done to compare the performance of the different fluorochromes in indirect flow microsphere assays using the $L X 100$ analyzer. PE is one of the brightest fluorescent molecules available as a reporter label. PE is a high molecular weight protein (MW - 240,000), and the streptavidin conjugate is bulkier than streptavidin labeled to small molecular weight fluorochromes potentially leading to slower reaction kinetics. Hence streptavidin labeled PE was tested in comparison with Cy3, Alexa 532 and Alexa 546. Results of these tests are shown in Table 4. It is clear that the no wash format showed higher nonspecific binding and lower signal to noise ratio. PE conjugates from two different manufacturers showed comparable results. About 5-fold reduction in signal and about 3-fold reduction in signal to noise were observed with streptavidin labeled Cy3, Alexa 532 and Alexa 546 compared with PE. Cy3 showed the brightest signal among the three small molecular weight fluorochromes.

Conclusions drawn from the above experiments are summarized in Table 5 for both direct or indirect, and also wash or no wash format assays. Direct assays with Cy3 in 
a no wash format gave good signal to noise ratio and washing steps improved the performance by two fold. It gave the best signal to noise ratio in only a single step reaction with the microspheres. However, the dynamic range of fluorescence intensity was found out to be less than when PE was used as reporter. Hence, this approach is the preferable one when Cy3 fluorescent labeling of the products is performed directly in a PCR amplification. The indirect, two step assay - first reaction of hybridization of the capture beads with biotinylated probe followed by the addition of streptavidin $\mp$ gave the best fluorescent intensity and very good signal to noise ratio. It also has broad dynamic range and hence useful for quantifying up to 3- to 4-Log concentrations of the product. Streptavidin PE can be used for the detection of PCR products in a direct binding assay if either a biotinylated primer, or biotinylated nucleotide tri-phosphates, are used during PCR amplification. This method can also be used in a competitive inhibition assay by hybridizing the complementary fluorescent probe and the unlabeled PCR product with the microspheres. This competitive inhibition method was used for the blind laboratory trial at LLNL for the detection of $\mathrm{Bg}$ and $\mathrm{Bt}$.

\section{6-plex oligonucleotide binding assay for $\mathrm{Bg}$, $\mathrm{Bt}$ and $\mathrm{Eh}$ probes}

A set of six probes was used for identifying $\mathrm{Bg}, \mathrm{Bt}$ and $\mathrm{Eh}$ specific nucleic acid sequences using the fluorescent microsphere assay. Carboxylated microspheres 011 , 022, 033, 045,065 and 078 were coated covalently with amino-labeled EhT, BgT, BtT, EhAT, and BgAT and BtAT oligonucleotides. The beads for this study were from the second batch of microspheres from Luminex, and thus were different from the beta microspheres used in earlier experiments. Biotinylated complementary probes and streptavidin PE were used in the indirect assay with washing steps. A dot plot from a LX 100 analysis of a 6-plex mixture is shown in Fig. 5. Specific binding of each complementary probe to its respective microsphere set was observed. The results of these 6-plex assays are shown in Fig. 6. Different probes showed varying levels of positive fluorescent intensity for the same amount of biotinylated complementary oligos. The variations observed may be due to the subtle differences in the microsphere sets, or differences in the coupling efficiency to the beads, or due to the nature of the complementary probe binding to the capture oligonucleotides. While there are some quantitative intensity differences among reporters, the results in Fig. 5 clearly show unambiguous identification of each of these reporters in a 6-plex analysis. 
Competitive oligonucleotide inhibition 3-plex fluorescent microsphere assay for the detection of $\mathrm{Bg}$ and $\mathrm{Bt}$ PCR amplified products for the laboratory trial conducted at LLNL

A competitive hybridization approach was developed to allow bead capture assays to be used for the identification of species specific PCR products resulting from multiplex PCR amplification reactions. With competitive hybridization, a fluorescent reporter oligo can hybridize with either its capture bead or with its PCR product. The reporter binds only to the beads yielding a bright bead fluorescence if the target PCR product is absent. If the target PCR product is present, however, some of the reporter hybridizes to the product reducing the beads fluorescence intensity.

The amine labeled oligonucleotides Eh 512T, Bg 238T and CRY IV 182AT were covalently coupled to three bead sets from a third batch of Luminex microspheres; bead sets $\mathrm{C} 152, \mathrm{C} 153$ and $\mathrm{C} 158$ respectively. This batch of microspheres was different from the earlier batches in their fluorescence classification intensities. They fall in a new bead map generated by the latest software upgrade. Some hardware changes were also made in the $L X 100$ to improve the reporter laser in the optical unit, and this required the production of a new batch of beads that were compatible with the new optics.

Our plan for the LLNL laboratory trial was to perform a 3-plex analysis of PCR products from the three bacterial species B.g., B.t., and E.h. Difficulties were encountered with the E.h. assay using the new bead set, however, so we limited the trial to a 2-pex assay for B.g. and B.t. Bead set C152 coated with oligonucleotide Eh 512T was retained to serve as a control bead set for the assays. Competitive inhibition assays were performed using 24 blind samples of PCR products, along with 4 known no template controls (NTC), an oligonucleotide only positive control, and negative no probe bead controls. Raw values of the median fluorescent intensity (MFI) of the three bead types for each of these samples are given in Table 6. The decrease in MFI from the NTC samples was calculated for each test sample, to determine if the sample was positive for amplification of $\mathrm{Bg}$ or $\mathrm{Bt}$. An arbitrary cut off value was set for the reduction of $\mathrm{MF}$ values of 60 for $\mathrm{Bg}$ beads and 100 for $\mathrm{Bt}$ beads below their NTC values to indicate a positive. The average of MFI of the NTC for Bg was 642 and Bt was 724 . Using this criterion, all of the 24 samples were identified correctly except for one NTC, once the samples were decoded and the true identity was revealed. A repeat test done on the following day also gave similar results. The false positive identification of $1 \mathrm{NTC}$ sample 
with a MFI of 590 for $\mathrm{Bg}$ beads and 561 for $\mathrm{Bt}$ beads resulted in an apparent $\mathrm{w}$ positive for both Bg and Bt instead of NTC.

The average MF values for each bead set was calculated for each decoded sample type, and the results are shown in Fig. 7. It is clear from the histogram that the positive samples showed significant reductions in MF values compared with the NTC controls, indicating a competitive inhibition of fluorescence due to the specific PCR amplification of $\mathrm{Bg}$ or $\mathrm{Bt}$ sequences in the sample. A strange fact was noted with the beads C158bBtAT. There was a significant reduction ( $50 \%$ ) in the fluorescence of the NTC samples compared to the oligo only control. This decrease may result from non-specific inhibition of fluorescence due to some components of the PCR mixture or amplification process in the NTC samples. However, this inhibition was reversed when there was $\mathrm{Bg}$ amplification in the sample.

MFI values of the 24 samples are grouped and the $\mathrm{Bg}$ specific fluorescence intensities are shown in Fig. 8. At least one sample identified as Bg + in Fig. 8 showed MFI close to the NTC samples and could have been missed and identified as negative if a stringent threshold value was set for the inhibition of fluorescence. MFl values of the samples grouped according to the Bt specific fluorescence intensities are shown in Fig. 9. It can be seen in Fig. 9 that one of the NTC and couple of samples identified as Bt + have MFls that are close to the NTCs and could have been misidentified by choosing different threshold values. Thus, although a few samples yielded ambiguous intensities, most samples could be clearly identified in the competitive assay. One important observation from Figs. 8 and 9 is that the intensity decrease of positive samples was the same for all starting concentrations of spores in the PCR amplification. This suggests that regardless of the template concentration, if PCR amplification occurs there will be sufficient PCR product for multiplex analysis.

\section{Summary}

Multiplex fluorescent microsphere assays were developed for the detection of $\mathrm{Bg}, \mathrm{Bt}$ and Eh specific oligonucleotide sequences. Fluorescent labeling methods were developed to allow these assays to be performed on the compact $L X 100$ flow cytometer with comparable detection sensitivity and specificity as seen with a large laboratory flow cytometer. Bead capture of fluorescently labeled reporter oligonucleotides was used to evaluate a number of parameters effecting assay performance. Parameters studied included oligo coupling to beads (carboxylate vs. 
avidin), fluorescent labeling of reporter oligos (direct vs. biotin-avidin), different fluorescent reporter dyes, and the utility of washing steps in sample preparation. Overall, these studies suggest that different assay approaches may be optimal for different applications. Indirect labeling with streptavidin PE and extensive washing, for example, yields bright fluorescent labeling and a wide dynamic range, but sample preparation is slow and complex. The reduced performance of directly labeled oligos may be acceptable for other applications where speed and simplicity of sample preparation are more important than precise quantification. To date we have successfully used these methods to observe sequence specific hybridization in a 6plex bead assay.

Methods have also been developed to allow bead capture assays to be used for the identification of species specific PCR products resulting from multiplex PCR amplification reactions. A competitive hybridization format was chosen where a fluorescent reporter oligo can hybridize with either its capture bead or with its PCR product. The reporter binds only to the beads yielding a bright bead fluorescence if the target PCR product is absent. If the target PCR product is present, however, some of the reporter hybridizes to the product reducing the bead fluorescent intensity. A preliminary test of this approach using $\mathrm{Bg}$ and Eh suggested that competitive hybridization could be detected using multiplex bead assays.

A blind laboratory trial was performed at LLNL to provide a benchmark of the performance of PCR product identification using a competitive bead assay. While a 3plex assay for $\mathrm{Bg}, \mathrm{Bt}$, and $\mathrm{Eh}$ was designed, the trial was reduced to two target agents due to problems with the Eh reagents. Analysis of the results from multiplex assays on 24 blind samples show a number of strengths and weaknesses of this approach. Strengths observed from the trial include the following: (1) Correct identifications were inferred from the data for 23 out of 24 samples; (2) Both species were correctly identified in mixtures even when the two templates differed in starting concentration; and (3) Comparable assay response was observed over the full range of spore concentrations from $10^{3}$ to $10^{6}$ spores per $\mathrm{ml}$. Limitations observed from the trial include the following: (1) The indirect labeling method used in this trial is relatively slow and complex; (2) The failure of the Eh reagent, and the marginal results obtained with some samples indicates further development is required to increase assay reliability. Research is currently in progress to increase the simplicity and reliability of multiplex PCR product identification. 
Table 1 Description and coding of the PCR product samples used in the blind multiplexed LLNL laboratory tests

\begin{tabular}{|c|c|c|}
\hline S.No & Sample Description & $\begin{array}{c}\text { Sample } \\
\text { Identification \# }\end{array}$ \\
\hline 1 & No template control & 8 \\
\hline 2 & No template control & 19 \\
\hline 3 & No template control & 20 \\
\hline 4 & No template control & 10 \\
\hline 5 & No template control & 3 \\
\hline 6 & No template control & 22 \\
\hline 7 & $\mathrm{Bg} 10^{6}$ & 5 \\
\hline 8 & $\mathrm{Bg} 10^{6}$ & 21 \\
\hline 9 & $\mathrm{Bg} 10^{4}$ & 9 \\
\hline 10 & $\mathrm{Bg} 10^{4}$ & 4 \\
\hline 11 & $\mathrm{Bg} 10^{3}$ & 24 \\
\hline 12 & $\mathrm{Bg} 10^{3}$ & 11 \\
\hline 13 & Bt $10^{6}$ & 6 \\
\hline 14 & Bt $10^{6}$ & 2 \\
\hline 15 & Bt $10^{4}$ & 1 \\
\hline 16 & Bt $10^{4}$ & 14 \\
\hline 17 & Bt $10^{3}$ & 16 \\
\hline 18 & Bt $10^{3}$ & 23 \\
\hline 19 & $\mathrm{Bg} 10^{6} \& \mathrm{Bt} 10^{6}$ & 15 \\
\hline 20 & $\mathrm{Bg} 10^{6} \& \mathrm{Bt} 10^{6}$ & 18 \\
\hline 21 & $\mathrm{Bg} 10^{6} \& \mathrm{Bt} 10^{4}$ & 12 \\
\hline 22 & $\mathrm{Bg} 10^{6} \& \mathrm{Bt} 10^{4}$ & 7 \\
\hline 23 & $\mathrm{Bg} 10^{4} \& \mathrm{Bt} 10^{6}$ & 17 \\
\hline 24 & $\mathrm{Bg} 10^{4} \& \mathrm{Bt} 10^{6}$ & 13 \\
\hline
\end{tabular}


Table 2 Summary of the experiments evaluating the effect of washing and comparative assessment of different fluorescent labels in Luminex 100 Analyzer

\begin{tabular}{|c|c|c|c|c|c|}
\hline \multirow{2}{*}{ Expt } & \multirow{2}{*}{ Bead } & \multirow{2}{*}{ Probe } & \multirow{2}{*}{ Wash } & Mean & Mean \\
\hline & & & & Positive & Other/No dye \\
\hline 1 & SAvidin & D-CY3 & No & 431.7 & 1.9 \\
\hline 2 & SAvidin & D-BDY & No & 500.6 & 4.5 \\
\hline 3 & SAvidin & D-CY3 & Yes & 577.9 & 1.2 \\
\hline 4 & SAvidin & D-BDY & Yes & 652 & 2 \\
\hline 5 & $\mathrm{COOH}$ & D-CY3 & No & 174.8 & 3 \\
\hline 6 & $\mathrm{COOH}$ & D-BDY & No & 305.3 & 4.9 \\
\hline 7 & $\mathrm{COOH}$ & D-CY3 & yes & 133.1 & 1.3 \\
\hline 8 & $\mathrm{COOH}$ & D-BDY & yes & 217.9 & 1.6 \\
\hline 9 & $\mathrm{COOH}$ & A-PE & no & 1371.4 & 17.8 \\
\hline 10 & $\mathrm{COOH}$ & A-PE & yes & 1485.2 & 5.6 \\
\hline 11 & $\mathrm{COOH}$ & A-PE2 & yes & 1549.5 & 5.1 \\
\hline 12 & $\mathrm{COOH}$ & A-Су3 & yes & 347.1 & 2.7 \\
\hline 13 & $\mathrm{COOH}$ & A-A546 & yes & 114 & 1.6 \\
\hline 14 & $\mathrm{COOH}$ & A-A532 & yes & 231.2 & 2.2 \\
\hline
\end{tabular}


Table 3 Comparison of the signal to noise with LumAvidin beads and Carboxylated beads in the direct binding assay and the effect of washing

\begin{tabular}{|c|c|c|c|c|c|}
\hline \multirow{2}{*}{ Bead Type } & \multirow{2}{*}{ Reporter Label } & \multicolumn{2}{|c|}{ Positive MFI } & \multicolumn{2}{c|}{ Signal / Noise } \\
\cline { 3 - 6 } & & No Wash & Wash & No Wash & Wash \\
\hline \multirow{2}{*}{ LumAvidin } & Cy3 & 431.7 & 577.9 & 36.8 & 66.3 \\
& BODIPY-TMRx & 500.6 & 652.0 & 17.6 & 45.7 \\
\hline \multirow{2}{*}{ COOH } & Cy3 & 174.8 & 133.1 & 11.8 & 16.0 \\
& BODIPY-TMRx & 305.3 & 217.9 & 12.7 & 22.1 \\
\hline
\end{tabular}


Table 4 Evaluation of different Streptavidin fluorescent labels in the indirect oligonucleotide assay using carboxylated microspheres

\begin{tabular}{|c|c|c|c|c|}
\hline $\begin{array}{c}\text { Bead } \\
\text { Type }\end{array}$ & $\begin{array}{c}\text { Streptavidin } \\
\text { Reporter } \\
\text { Fluorescent } \\
\text { Label }\end{array}$ & $\begin{array}{c}\text { Positive } \\
\text { MFI }\end{array}$ & $\begin{array}{c}\text { Signal / } \\
\text { Noise }\end{array}$ & $\begin{array}{c}\text { Negative/No } \\
\text { Reporter label } \\
\text { (Non specific } \\
\text { binding) }\end{array}$ \\
\hline COOH & PE (No wash) & 1371 & 12.2 & 17.8 \\
& Wash & 1485 & 50.3 & 5.6 \\
& PE & 1550 & 55.2 & 5.1 \\
& Cy3 & 347 & 20.3 & 2.7 \\
& Alexa 532 & 231 & 15.7 & 2.2 \\
& Alexa 546 & 114 & 10.7 & 1.6 \\
\hline
\end{tabular}


Table 5 Summary of the best conditions for labeling and the dye for oligonucleotide microsphere assay

\begin{tabular}{|c|c|c|c|c|}
\hline $\begin{array}{c}\text { Labeling } \\
\text { Steps }\end{array}$ & $\begin{array}{c}\text { Wash } \\
\text { Step }\end{array}$ & $\begin{array}{c}\text { Dye } \\
\text { Label }\end{array}$ & $\begin{array}{c}\text { Positive } \\
\text { Intensity }\end{array}$ & $\begin{array}{c}\text { Positive / } \\
\text { Negative }\end{array}$ \\
\hline 1-step & Yes & Cy3 & 578 & 66 \\
1-step & No & Cy3 & 432 & 37 \\
2-step & Yes & SAv-PE & 1550 & 55 \\
\hline
\end{tabular}


Table 6 Raw median fluorescent intensity values microspheres in the 3-plex competitive oligonucleotide inhibition microsphere assay for the detection of $\mathrm{Bg}$ and $\mathrm{Bt} P C R$ amplified products

\begin{tabular}{|c|c|c|c|c|c|}
\hline S. No. & C152aEhT & C153aBgAT & C158aBtAT & Test Inference & $\begin{array}{c}\text { Actual } \\
\text { Sample Identity }\end{array}$ \\
\hline 1 & 12 & 691 & 573.5 & Bt & Bt \\
\hline 2 & 13 & 633 & 518.5 & Bt & $\mathrm{Bt}$ \\
\hline 3 & 15 & 627.5 & 776 & NTC & NTC \\
\hline 4 & 14 & 462 & 1624.5 & $\mathrm{Bg}$ & $\mathrm{Bg}$ \\
\hline 5 & 30 & 470 & 1713.5 & $\mathrm{Bg}$ & $\mathrm{Bg}$ \\
\hline 6 & 14 & 631 & 480 & $\mathrm{Bt}$ & $\mathrm{Bt}$ \\
\hline 7 & 11.5 & 507 & 411 & $\mathrm{Bg} \mathrm{Bt}$ & $\mathrm{Bg} \mathrm{Bt}$ \\
\hline 8 & 15 & 627 & 635 & NTC & NTC \\
\hline 9 & 13 & 483 & 1647.5 & $\mathrm{Bg}$ & $\mathrm{Bg}$ \\
\hline 10 & 16 & 590 & 561 & $\mathrm{Bg} \mathrm{Bt}$ & NTC \\
\hline 11 & 12 & 396 & 1705 & $\mathrm{Bg}$ & $\mathrm{Bg}$ \\
\hline 12 & 14 & 465.5 & 381 & $\mathrm{Bg} \mathrm{Bt}$ & $\mathrm{Bg} \mathrm{Bt}$ \\
\hline 13 & 15 & 413 & 285 & $\mathrm{Bg} \mathrm{Bt}$ & $\mathrm{Bg} \mathrm{Bt}$ \\
\hline 14 & 13.5 & 673.5 & 541.5 & Bt & Bt \\
\hline 15 & 14.5 & 477 & 442 & $\mathrm{Bg} \mathrm{Bt}$ & $\mathrm{Bg} \mathrm{Bt}$ \\
\hline 16 & 13 & 640.5 & 508.5 & $\mathrm{Bt}$ & $\mathrm{Bt}$ \\
\hline 17 & 15.5 & 436 & 278 & $\mathrm{Bg} \mathrm{Bt}$ & $\mathrm{Bg} \mathrm{Bt}$ \\
\hline 18 & 98.5 & 571 & 589 & $\mathrm{Bg} \mathrm{Bt}$ & $\mathrm{Bg} \mathrm{Bt}$ \\
\hline 19 & 16 & 641 & 673 & NTC & NTC \\
\hline 20 & 70.5 & 710 & 771.5 & NTC & NTC \\
\hline 21 & 13.5 & 366 & 1654.5 & $\mathrm{Bg}$ & $\mathrm{Bg}$ \\
\hline 22 & 17 & 642 & 821 & NTC & NTC \\
\hline 23 & 13 & 638 & 508 & Bt & Bt \\
\hline 24 & 25 & 458.5 & 1676.5 & $\mathrm{Bg}$ & $\mathrm{Bg}$ \\
\hline 25 & 12 & 629 & 772 & Known- & NTC Control \\
\hline 26 & 18 & 536 & 765.5 & Known- & NTC Control \\
\hline 27 & 13 & 601 & 754 & Known- & NTC Control \\
\hline 28 & 12 & 561 & 708.5 & Known- & NTC Control \\
\hline 29 & 14 & 574 & 1470.5 & Known + & Oligo Positive Control \\
\hline 30 & 13 & 529 & 1437.5 & Known + & Oligo Positive Control \\
\hline 31 & 13 & 11 & 9 & Known & Blank \\
\hline 32 & 14 & 12.5 & 12 & Known & Blank \\
\hline 33 & 9 & 8 & 7 & Known & Test bds \\
\hline 34 & 9 & 9 & 8 & Known & Test Bds \\
\hline
\end{tabular}




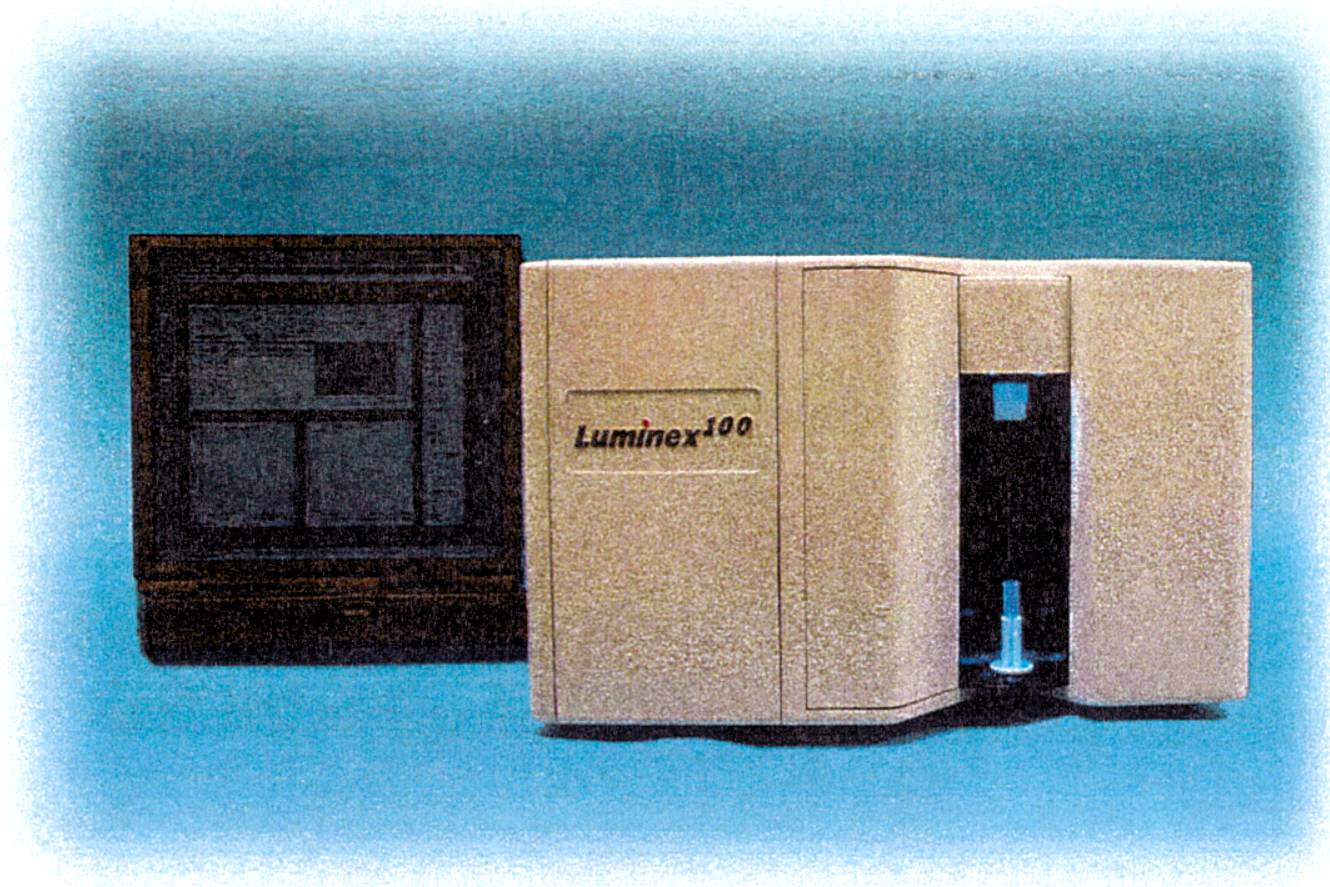

Fig. 1 Luminex 100 Analyzer 


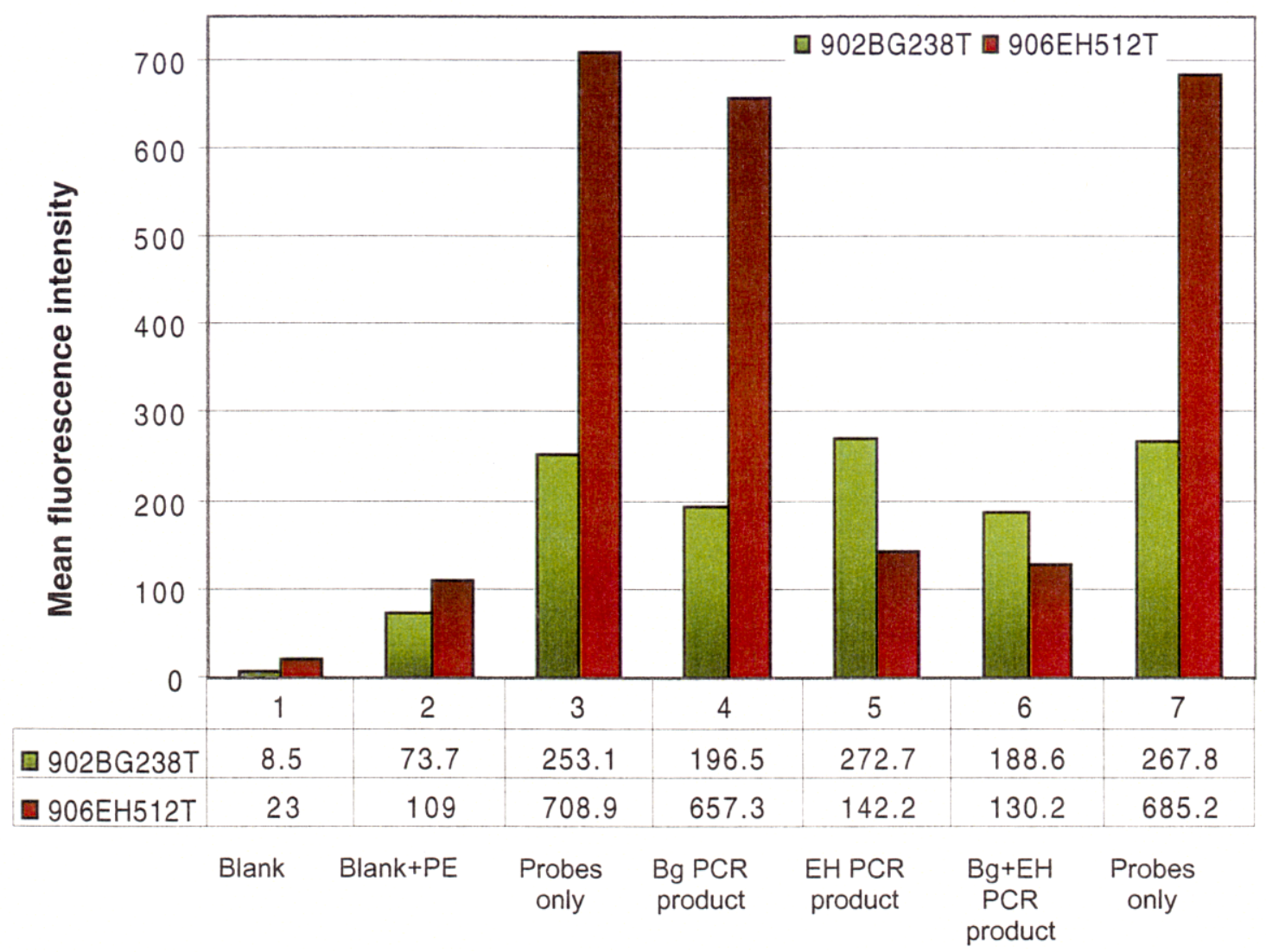

Fig. 2 Duplex flow microsphere oligonucleotide inhibition assay for Bg and Eh PCR product identification 


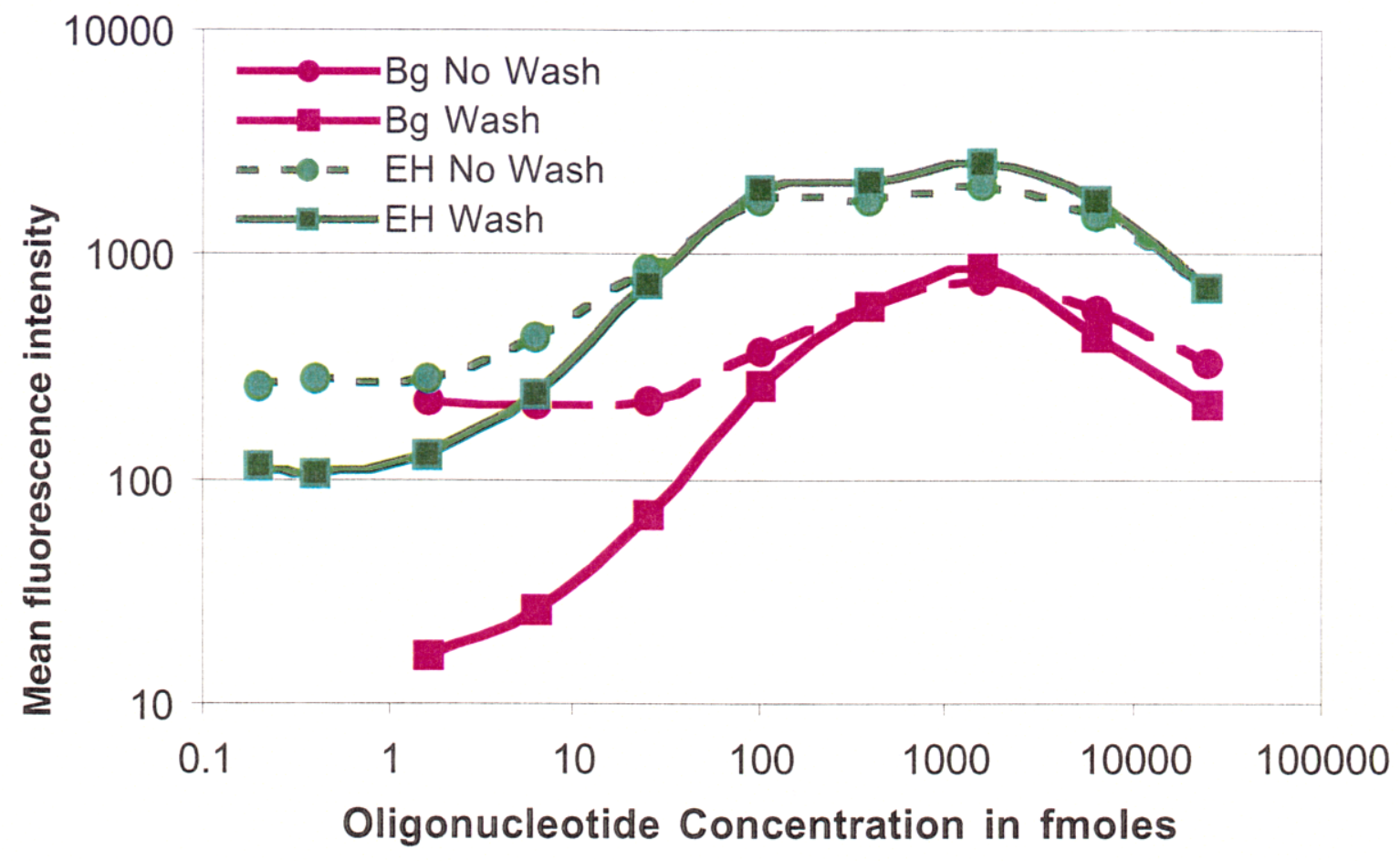

Fig. 3 Evaluation of washing on $\mathrm{Bg}$ and Eh specific fluorescent probes binding with specific capture-oligonucleotide on microspheres 


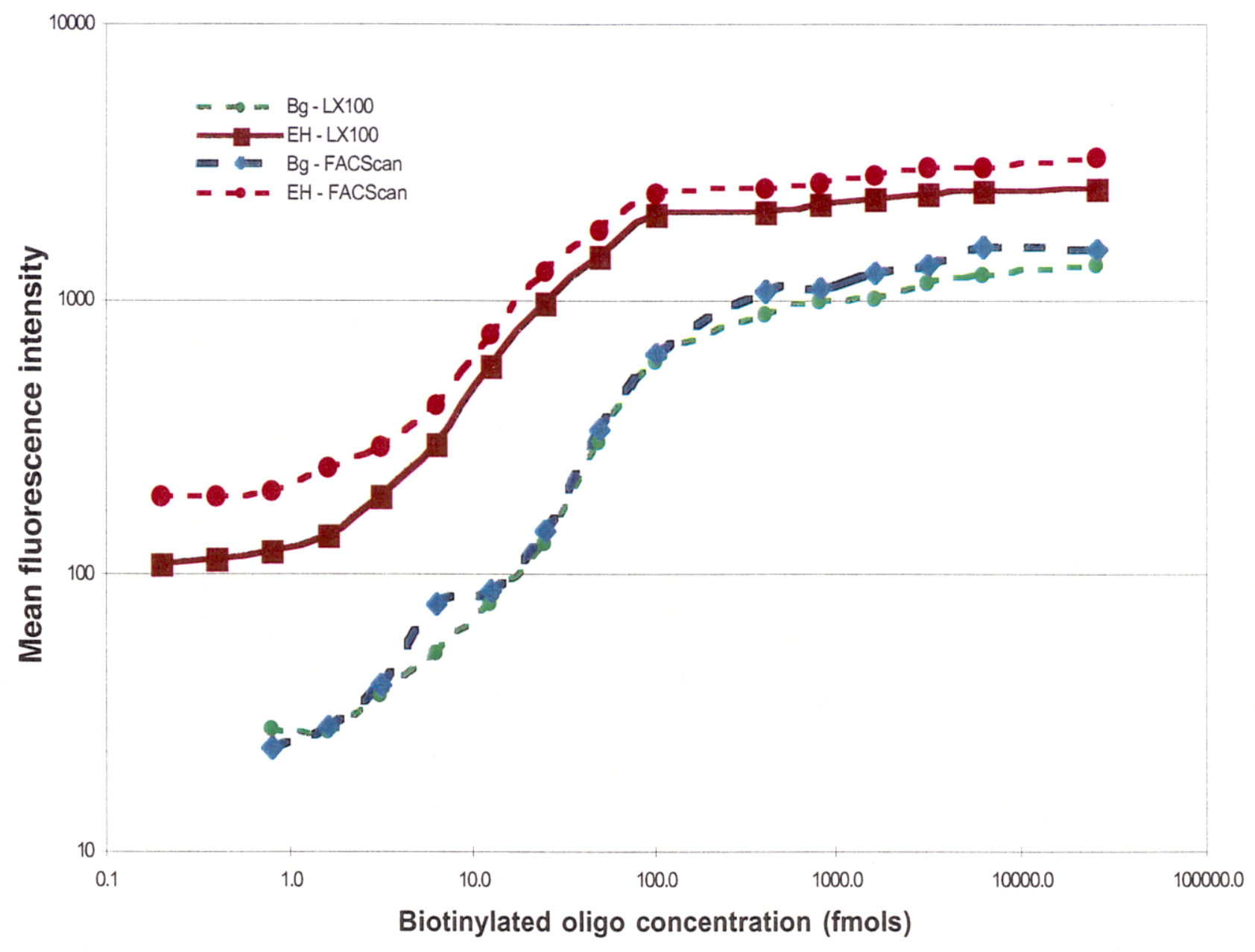

Fig. 4 Comparison of the microsphere analysis with Luminex 100 Analyzer and conventional BD FACScan flow cytometer 


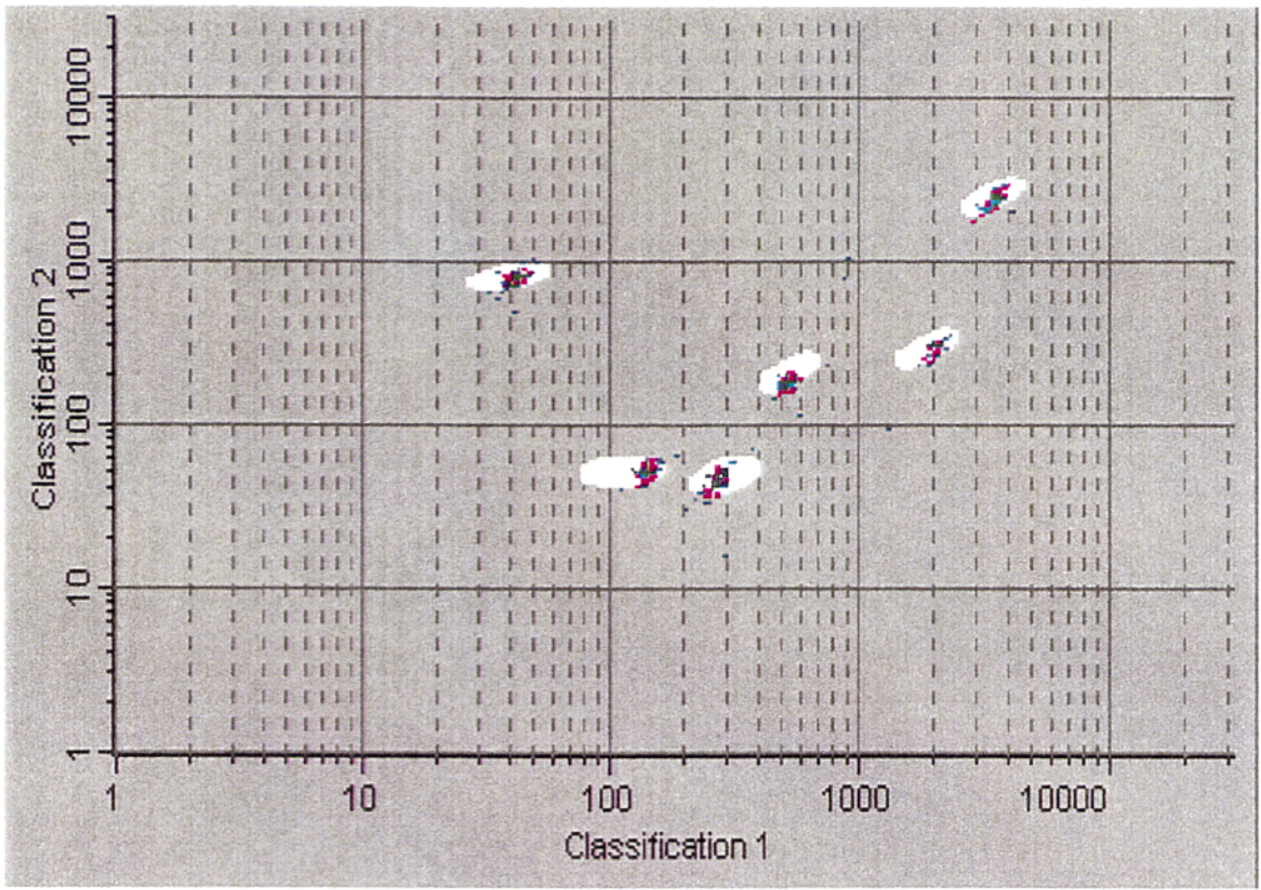

Fig. 5 Dot plot showing 6-plex microsphere mixture in fluorescent classification 1 and classification 2 in LX 100 Flow Analyzer 


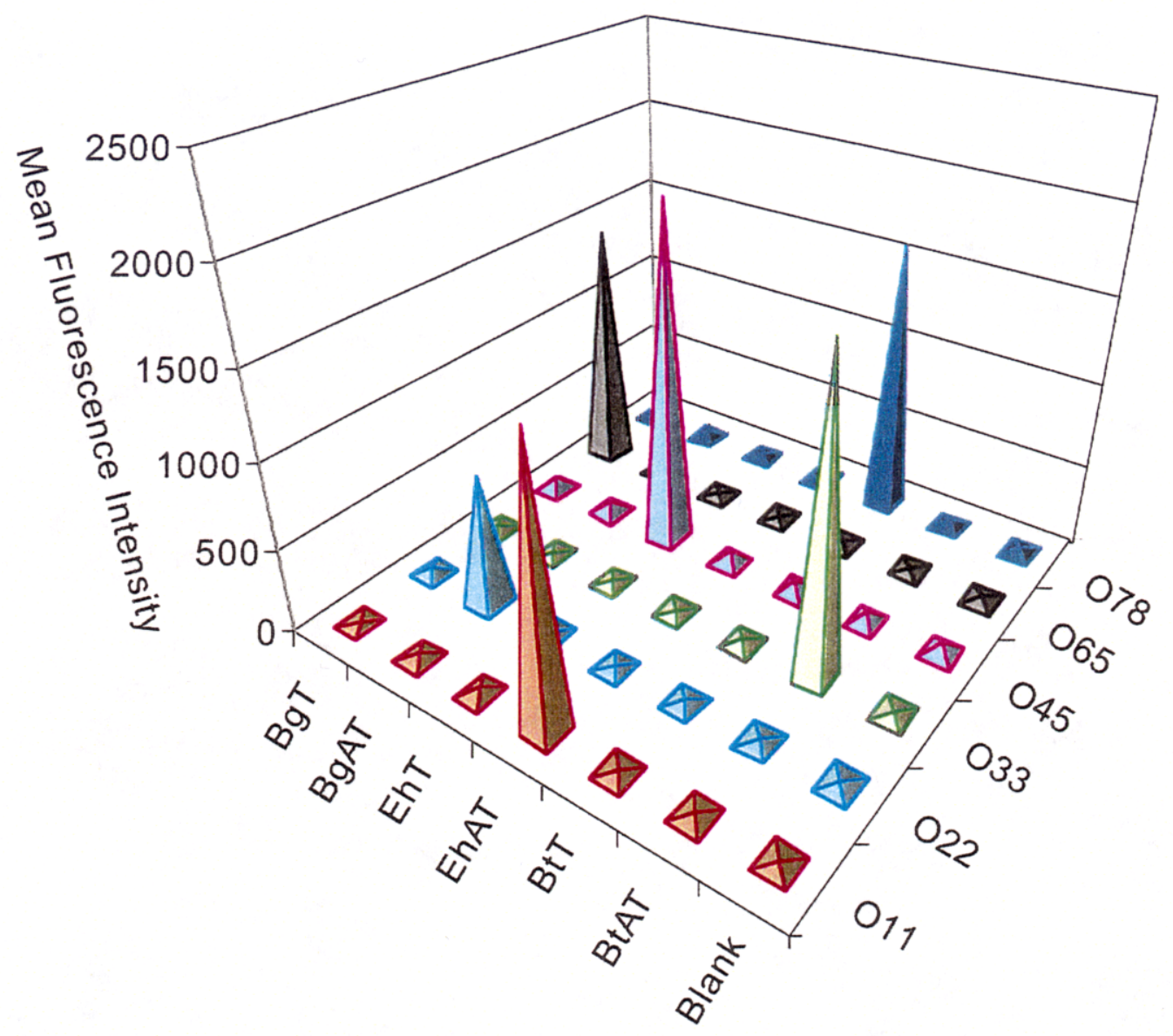

Fig. 6 Fluorescence oligonucleotide multiplex assay detection of nucleic acid sequences specific for $\mathrm{Bg}, \mathrm{Bt}$ and Eh using 6-plex microsphere mixture 


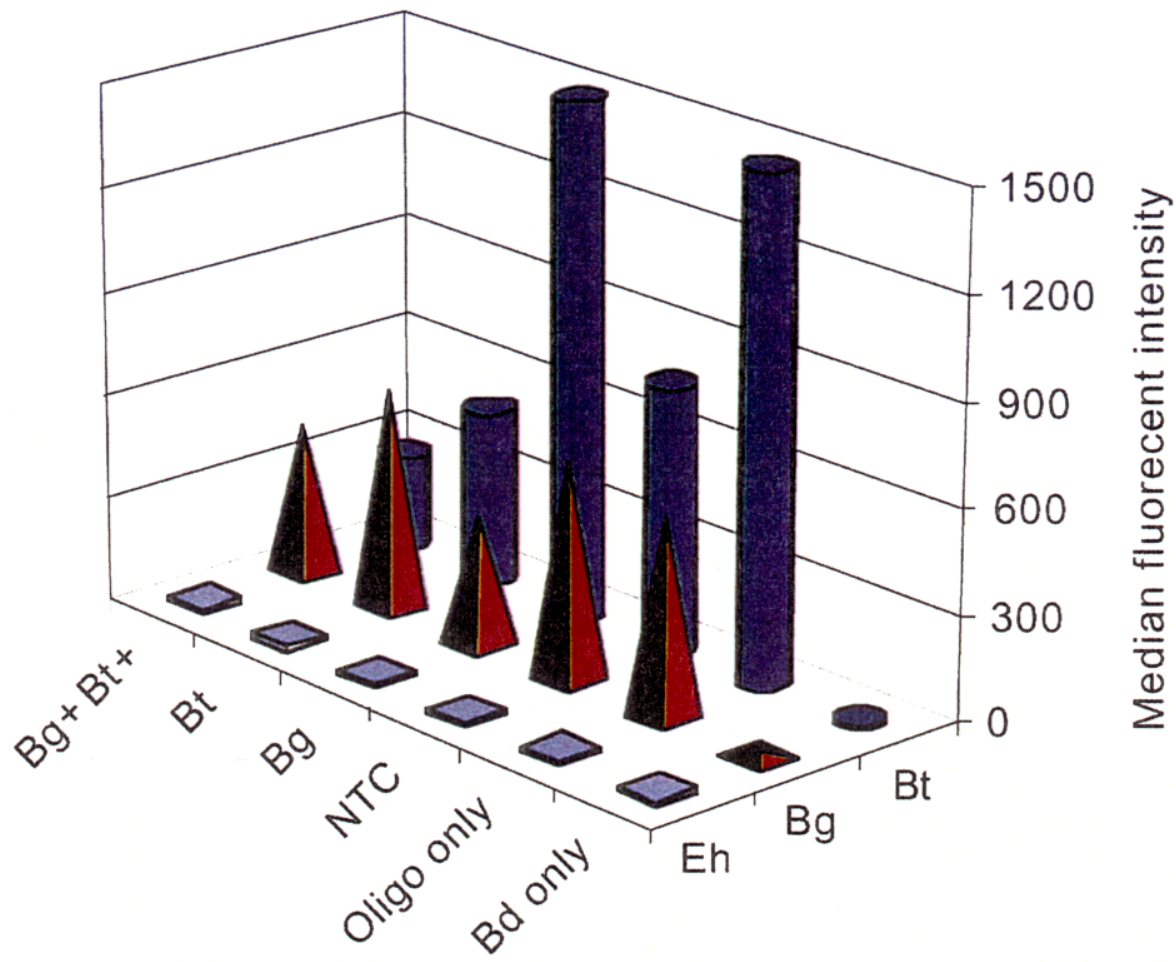

Fig. 7 Average of median fluorescent intensity of 3-plex microspheres coated with $\mathrm{Bg}, \mathrm{Bt}$ and Eh specific oligonucleotides in competitive inhibition assay 


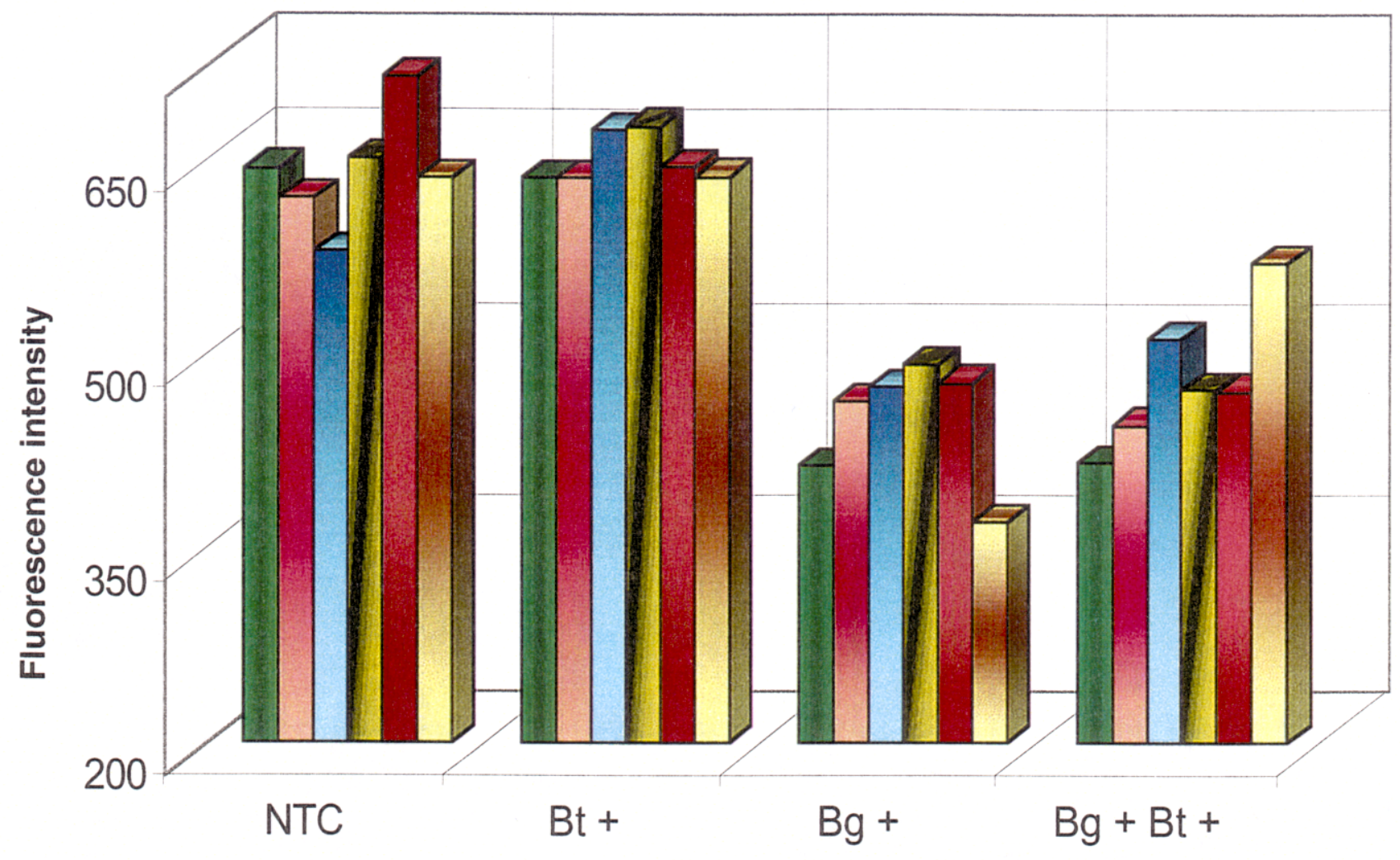

Fig. 8 Histogram of median fluorescence intensities of the 24 blind samples tested for the specific binding of PCR amplified products to Bg specific capture oligonucleotide coated microspheres 


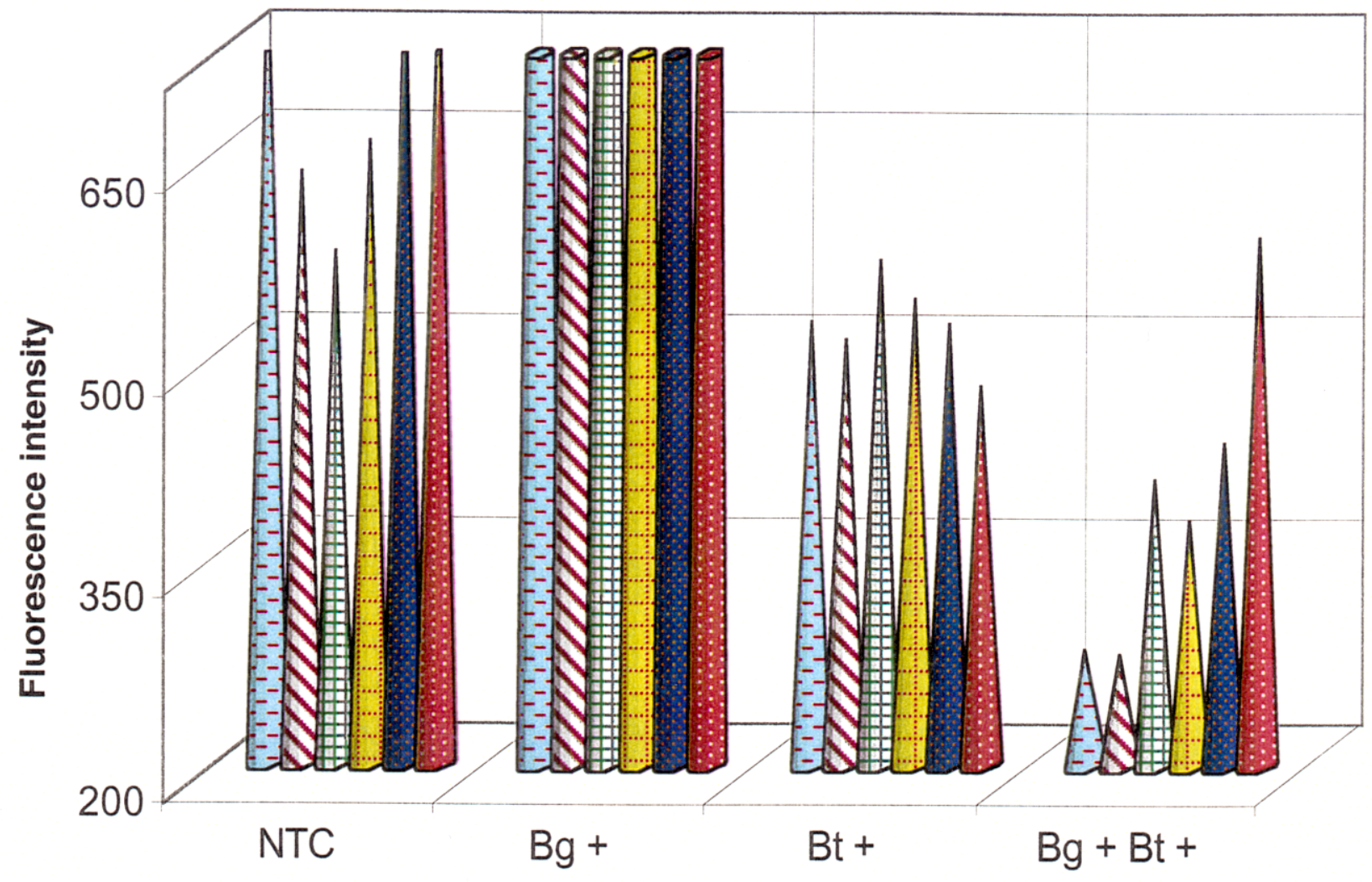

Fig. 9 Histogram of median fluorescence intensities of the 24 blind samples tested for the specific binding of PCR amplified products to Bt specific capture oligonucleotide coated microspheres 